\title{
The protective effect of carbenoxolone on gap junction damage in the hippocampal CA1 area of a temporal lobe epilepsy rat model
}

\author{
Yi Shu, Can Zhu, Min Zeng, Qiong Zhan, Zhiping Hu, Xiaomei Wu \\ Department of Neurology, The Second Xiangya Hospital, Central South University, Changsha 410011, China \\ Contributions: (I) Conception and design: X Wu; (II) Administrative support: Z Hu; (III) Provision of study materials or patients: Q Zhan; (IV) \\ Collection and assembly of data: Y Shu, C Zhu, M Zeng; (V) Data analysis and interpretation: Y Shu, X Wu; (VI) Manuscript writing: All authors; (VII) \\ Final approval of manuscript: All authors. \\ Correspondence to: Xiaomei Wu. Department of Neurology, The Second Xiangya Hospital, Central South University, No. 139 Middle Renming Road, \\ Changsha 410011, China. Email: wuxiaomei0923@csu.edu.cn.
}

\begin{abstract}
Background: Astrocytes are one of the most important types of neural cells in the central nervous system (CNS). Dysfunctional gap junction (GJ) communication could play an underlying role in epileptogenesis. Carbenoxolone (CBX) is a conventional chemical GJ blocker, and its target is connexin 43 (Cx43). Previous studies have shown that CBX can inhibit status epilepticus (SE) and spontaneous epileptic seizures (SESs). However, there is little information about the direct interaction between CBX and Cxs in temporal lobe epilepsy (TLE).
\end{abstract}

Methods: The behavior of epileptic rats was observed. Moreover, micromorphological changes in the hippocampal cornu ammonis 1 (CA1) area of epileptic rats following CBX injection were determined through transmission electron microscopy (TEM). To illustrate the possible mechanism of these changes, the Western blot method was used.

Results: After the injection of CBX, the seizure frequency, seizure duration, latency period to the first instance of SES, SESs behavioral score according to a scoring system developed by Velíšková and microstructures in the CA1 area were shown to be improved 60 days after SE by TEM. Furthermore, the dynamic expression patterns of $\mathrm{Cx} 43$ and $\mathrm{Cx} 43$ phosphorylated at Ser368 continuously declined after the injection of CBX until 60 days after SE.

Conclusions: CBX may contribute to the improvement of GJ dysfunction during epileptogenesis in the hippocampal CA1 area in a TLE rat model.

Keywords: Epilepsy; astrocyte; gap junction (GJ); connexin 43 (Cx43); carbenoxolone (CBX)

Submitted Jul 25, 2019. Accepted for publication Oct 10, 2019.

doi: $10.21037 /$ atm.2019.11.04

View this article at: http://dx.doi.org/10.21037/atm.2019.11.04

\section{Introduction}

Temporal lobe epilepsy (TLE) is the most clinically prevalent type of partial complex seizure. Although there is an increasing number of new antiepileptic drugs, $20-30 \%$ of patients still have implacable seizures (1). To treat patients with implacable seizures, it is critical to clarify the potential mechanisms underlying epileptogenesis.

Astrocytes are the most resourceful glial cell type in the central nervous system (CNS). They play a crucial role in the mechanical and metabolic support of neurons by interacting with neuronal synapses via the uptake of neurotransmitters the delivery of receptor-mediated $\mathrm{Ca}^{2+}$ signaling and the subsequent release of gliotransmitters (2). An increasing number of studies have found that astrocyte dysfunction could contribute to the etiology and progression of epilepsy (3). In the hippocampus, approximately $60 \%$ of all synapses are enwrapped by astrocytes (4). The perisynaptic astrocyte membrane contains various ion channels and transporters, and astrocyte disruption may influence the homeostasis of synaptic transmission in 
even the entire neural network through the release of gliotransmitters and neuromodulators such as glutamate, ATP, and D-serine $(5,6)$.

The gap junction (GJ) is an important kind of ion channel involved in the transmission of information through neuron-glial or glial-glial interactions. Each GJ consists of two hemichannels (termed connexons), each of which is composed of six subunits of proteins called connexins (Cxs). Inflammation-induced disturbance and dysfunction of astrocytic GJ coupling are a critical step in epileptogenesis (7). Seizures can be blocked by GJ blockers, and GJ openers exacerbate seizure activity. In the adult brain, the astrocytic GJ components are mainly the connexin $43(\mathrm{Cx} 43)$ protein and the $\mathrm{Cx} 30$ protein (8). Cell type-specific deletion of Cxs in mice leads to the generation of spontaneous epileptiform activity (9).

Carbenoxolone (CBX) is a common chemical blocker of GJs that is specifically used as a $\mathrm{Cx} 43$ antisense oligonucleotide (Cx43 ASODN) and peptide mimetic. Ventura-Mejía et al. found that CBX decreased the mean number of fast ripples (FRs) and the number of oscillation cycles per FR event in the hippocampi of rats with spontaneous epileptic seizure (SES) (10). Kékesi et al. reported that CBX prevented recurrent seizurelike activity and induced unsynchronized $\mathrm{Ca}^{2+}$ transients associated with the decreasing incidence of epileptiform discharges in a low $\mathrm{Mg}^{2+}$ in vitro epilepsy model (11). More importantly, Sefil et al. found that the seizure latency, seizure frequency, spike duration and Racine scale score declined in a dose-dependent manner in pentylenetetrazole (PTZ) kindling rats injected with CBX (12). Chen et al. determined that CBX pretreatment or treatment significantly reduced $\mathrm{Cx} 43$ expression, inhibited glial cell proliferation around a cortical focus and attenuated the seizure degree of posttraumatic epilepsy (13). Recently, it found that CBX could inhibit FRs by decreasing $\mathrm{Cx} 43$ expression in a lithiumpilocarpine-induced rat model of TLE (9); however, how CBX deeply effect on $\mathrm{Cx} 43$ and the GJ microstructures of this rat model needs to be more explored.

In the present study, we used CBX as a drug blocker to investigate the expression of the $\mathrm{Cx} 43$ protein in a rat model of TLE. Furthermore, we observed morphological changes in the hippocampal cornu ammonis 1 (CA1) area and the behavior of epileptic rats to illustrate the potential mechanism by which Cxs mediate astrocytic GJ communication during epileptogenesis.

\section{Methods}

\section{Animals and models}

Healthy male and specific pathogen-free (SPF) SpragueDawley rats (SD, 180-200 g, 6-week-old) were purchased from SLAC Laboratory Animal Co., Ltd. (Shanghai, China) and housed in the Experimental Animal Center of Central South University (Changsha, China). Three rats were housed per cage maintained at a temperature of $20 \pm 2{ }^{\circ} \mathrm{C}$ with $50-60 \%$ humidity under a regular 12 -h light-dark cycle and given free access to water and food. The animals were cared for in accordance with the National Institutes of Health Guide for the Care and Use of Laboratory Animals, and the Animal Ethics Committee of Central South University approved their use. Only the minimum number of animals necessary for this study was used. Care was taken to avoid unnecessary suffering or pain during animal experimentation.

During this study, the rats $(\mathrm{n}=80)$ were divided into a status epilepticus (SE) group or a CBX-injected group (CBX group). The rats were injected with lithiumpilocarpine (lithium chloride, $125 \mathrm{mg} / \mathrm{kg}$, i.p., SigmaAldrich, Saint Louis, MO, USA; pilocarpine, $20 \mathrm{mg} / \mathrm{kg}$, i.p., Alfa Aesar, MA, USA), which successfully induced $\mathrm{SE}$; there were 63 rats survived. After lithiumpilocarpine was administered, the behaviors of the rats were observed. Convulsion severity was evaluated using Racine's classification ranging from stage $0-5$. The rats with no seizures were classified as below Racine stage 4 and injected with pilocarpine $(10 \mathrm{mg} / \mathrm{kg}$, i.p.) every $30 \mathrm{~min}$ up to 6 times. SE was defined as continuous seizures lasting at least $30 \mathrm{~min}$. Only rats classified as Racine stage 4 to stage 5 that fulfilled the SE criterion were used in this study. The maximum dose of pilocarpine was $75 \mathrm{mg} / \mathrm{kg}$. All rats were administered $10 \%$ chloral hydrate $(\mathrm{CH}, 300 \mathrm{mg} / \mathrm{kg}$, i.p., the Second Xiangya Hospital, Changsha, China) after $30 \mathrm{~min}$ to terminate SE. Additional $\mathrm{CH}$ was given if seizures were not attenuated sufficiently or recurred within $10 \mathrm{~min}$ after the first $\mathrm{CH}$ injection. Agematched rats in a control group (Con group) were injected with a similar amount of sterile saline instead of lithium chloride and pilocarpine. Twenty-four hours later, 9 of the surviving rats were randomly chosen and sacrificed as the SE-24h subgroup. Then, 9 rats were sacrificed at 7, 15, 30 and 60 days after the onset of SE as the SE-7d, SE-15d, SE30d, and SE-60d subgroups. The rats in the CBX group were administered CBX (20 mg/kg, i.p.) once per day beginning 
7 days after SE development and randomly sacrificed at 15, 30 and 60 days after the onset of SE as the CBX-15d, CBX$30 \mathrm{~d}$, and CBX-60d subgroups. Equal number of rats in the Con group were also sacrificed. Of the surviving rats, all rats in the SE-30d and SE-60d subgroups and 7 rats in the CBX-15d and CBX-60d subgroups appeared to undergo SESs, as determined by using video monitoring. The SESs typically consisted of periods of epileptic behaviors, such as rhythmic nodding, chewing, and unilateral or bilateral forelimb clonus lasting 1-3 min according to the Velîśková scoring system (14). The frequency of SESs among the different rats varied from $0-5$ times per day beginning 30 days after $\mathrm{SE}$.

\section{Behavioral recording}

The behaviors of rats picked up randomly were recorded on video without interruption from 30 to 60 days after SE ( $\mathrm{n}=6$ in the SE subgroup and the CBX subgroup). The number of seizures over 30 days and the seizure duration (in seconds) were recorded. At this stage, the fur of all of the rats was healthy. The rats had free access to food and water, and no rats died. At the end of the experiment, the rats used for behavioral observation were sacrificed and buried out of respect.

\section{Transmission electron microscopy (TEM)}

Anesthetized rats were conventionally perfused through the left ventricle with $4 \%$ paraformaldehyde ( $n=3$ in each subgroup). Tissues from three individual rat brains from each subgroup were prepared for TEM. The hippocampal tissue was then fixed in $2.5 \%$ glutaraldehyde in $0.1 \mathrm{~mol} / \mathrm{L}$ cacodylic acid buffer at $\mathrm{pH} 7.3$ for at least $24 \mathrm{~h}$ and postfixed in $2 \%$ osmic acid for $2 \mathrm{~h}$. Next, the fixed brain tissue was dehydrated in a graded ethanol series $(50 \%, 70 \%, 90 \%$ and $100 \%) 3$ times for 10 min each per level. The tissue was then soaked in a mixture of epoxy resin and acetone $(1: 1, \mathrm{v} / \mathrm{v})$ for $24 \mathrm{~h}$ at $37^{\circ} \mathrm{C}$, followed by embedding for $24 \mathrm{~h}$ at $60^{\circ} \mathrm{C}$ to harden an embedding mixture of Epon 812, dodecenyl succinic anhydride, methyl nadic anhydride, and dimethylaminomethyl phenol. The embedded tissue was then cut into semithin sections and stained with toluidine blue to select the CA1 area as the region of interest (ROI). The slices were then cut into ultrathin sections at a thickness of 500 angstroms by an LKB-III ultramicrotome (LKB, Sweden), mounted on copper grids, and doublestained with uranyl acetate and plumbous nitrate. Specimens were observed and photographed under a Hitachi HT-7700 transmission electron microscope (Hitachi, Japan).

\section{Western blotting}

The bilateral hippocampi of the rats ( $\mathrm{n}=6$ in each subgroup) were homogenized on ice in RIPA buffer (Beyotime, China) supplemented with the protease inhibitor PMSF (Solarbio, China) and the phosphatase inhibitor PhosSTOP (Roche, Switzerland). Total protein concentrations were determined with a modified bicinchoninic acid (BCA) assay. Proteins were separated by electrophoresis on $10 \%$ polyacrylamide gels and then transferred onto PVDF membranes at $300 \mathrm{~mA}$ for $2 \mathrm{~h}$ at $4{ }^{\circ} \mathrm{C}$ to separate the $\mathrm{Cx} 43$ and $\mathrm{p}-\mathrm{Cx} 43$ proteins. The membrane was blocked with $5 \%$ dried skim milk in TBS buffer $(\mathrm{pH} 7.5,50 \mathrm{mM}$ Tris$\mathrm{HCl}, 150 \mathrm{mM} \mathrm{NaCl}$ ) containing $0.1 \%$ Tween-20 (TBST) for $1 \mathrm{~h}$ at room temperature (RT). Blots were probed with primary antibodies against $\mathrm{Cx} 43$ (rabbit polyclonal Cx43 antibody, $43 \mathrm{kDa}, 1: 300$, Invitrogen, USA) and mouse $\beta$-actin (43 kDa, 1:1,000, Beyotime) overnight at $4{ }^{\circ} \mathrm{C}$. After washing with TBST, the membranes were incubated for 45-60 min at RT with a secondary anti-rabbit antibody (1:3,000, Proteintech Group, USA) conjugated to horseradish peroxidase and developed using an enhanced chemiluminescence system (Thermo, USA). The relative optical densities (RODs) of the specific bands visible on the images scanned by a Bio-Rad GelDoc 2000 imaging system (Bio-Rad, USA) were measured by the image analysis software Image Lab 3.0 (Bio-Rad, USA). Densities were quantified and normalized to the density of $\beta$-actin.

\section{Statistical analysis}

The results are presented as the mean \pm standard deviation (SD). All data were analyzed and made into bar graphs with GraphPad Prism 5.01 software. The significance of differences in $\mathrm{Cx} 43$ and $\mathrm{p}-\mathrm{Cx} 43$ protein expression at each time point was determined by one-way analysis of variance (ANOVA). Differences in the behavior patterns between the SE group and the CBX group were analyzed using an unpaired $t$-test. Statistical significance was indicated by $\mathrm{P}<0.05$.

\section{Results}

\section{The effect of CBX on rat behavioral patterns}

Starting 30 days after the initiation of SE, the behavior 
A

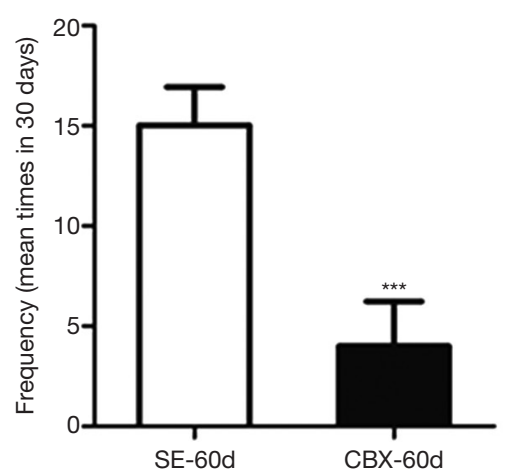

C

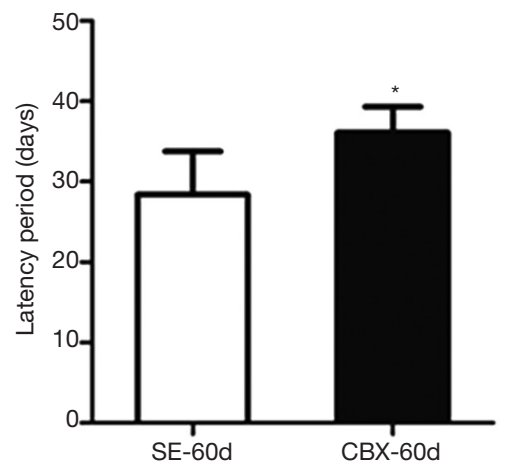

B
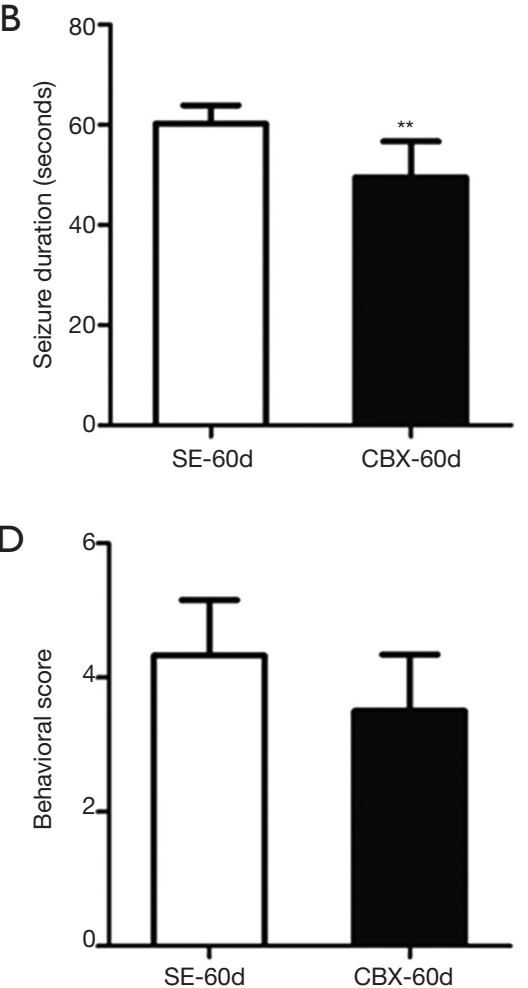

Figure 1 Characteristics of SESs in the SE-60d group and the CBX-60d group. (A) The bar graph shows the average frequency of SESs in the SE-60d group and the CBX-60d group. The average frequency was lower in the CBX-60d group; (B) the bar graph shows the average seizure duration in the SE-60d group and the CBX-60d group. The average seizure duration was lower in the CBX-60d group; (C) the bar graph shows the average seizure latency period in the SE-60d and CBX-60d groups. The average seizure duration was longer in the CBX-60d group; (D) the bar graph shows the average behavioral score in the SE-60d and CBX-60d groups. There was no difference in the average behavioral score between in the two groups. Values are the mean $\pm \mathrm{SD}$. Compared to $\mathrm{SE}-60 \mathrm{~d}:{ }^{* * *}, \mathrm{P}<0.001,{ }^{* *}, \mathrm{P}<0.01,{ }^{*}, \mathrm{P}<0.05$. SES, spontaneous epileptic seizure; SE, status epilepticus; CBX, carbenoxolone; SD, standard deviation.

patterns of the rats were monitored by video. The behavior of rats with SESs was characterized by unilateral or bilateral forelimb clonus with or without standing and falling. Classical tonic-clonic seizures were not observed. We compared the seizure frequency (mean number of seizures in 30 days), seizure duration (seconds), latency period to the first SES (days) and behavioral score according to the Velišková scale in the SE-60d group and the CBX-60d group in the chronic phase of seizure activity. The seizure frequency was lower in the CBX-60d group than in the SE60d group (SE-60d group: $15.02 \pm 1.92$ per month; CBX-60d group: $4.00 \pm 2.24$ per month; $\mathrm{n}=6, \mathrm{P}<0.001$; Figure $1 A$ ). The seizure duration was also lower in the CBX-60d group than in the SE-60d group (SE-60d group: 60.20 $\pm 3.7 \mathrm{~s}$; CBX-60d group: 49.60 $\pm .12 \mathrm{~s} ; \mathrm{n}=6, \mathrm{P}<0.01$; Figure $1 B$ ). Furthermore, the animals in the CBX-60d group showed longer latency periods to the first SES than those in the SE-60d group
(SE-60d group: $28.39 \pm 5.40$ days; CBX-60d group: $36.12 \pm 3.18$ days; $\mathrm{n}=6, \mathrm{P}<0.05$; Figure $1 C$ ). However, the behavioral scores were not significantly different between the two groups (SE-60d group: $4.33 \pm 0.82$; CBX-60d group: $3.50 \pm 0.84 ; \mathrm{n}=6, \mathrm{P}>0.05$; Figure $1 D$ ).

\section{The effect of $C B X$ on the microstructure of the CA1 area in the rat hippocampus}

The hippocampal cells were arranged in alignment and exhibited a complete and normal ultrastructure in the Con group (Figure 2A,B). The neuronal nuclei were round with an uninterrupted nuclear membrane. The mitochondria and endoplasmic reticulum (ER) were obvious in the cytoplasm. Furthermore, the subcellular structures of astrocytes were integrated, which had round or oval nuclei. The heterochromatin in the nuclei was present as a well- 

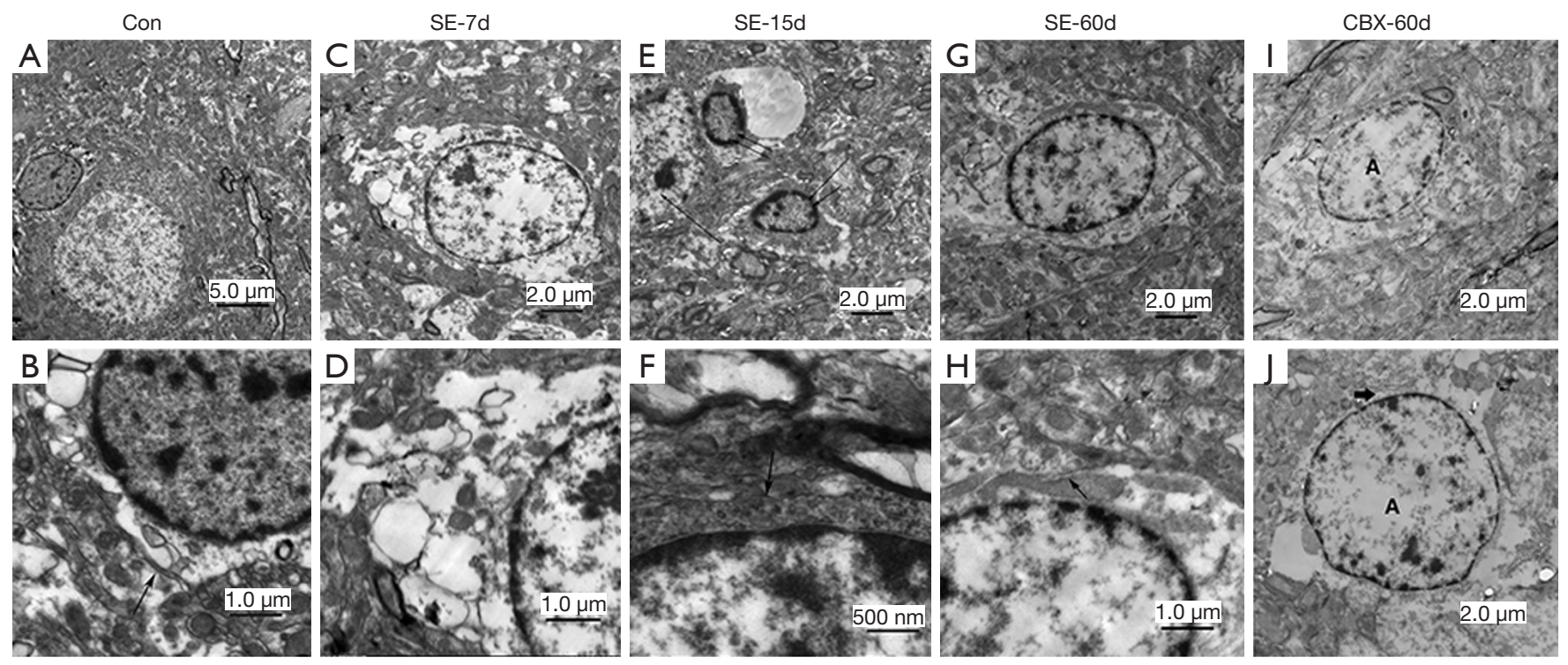

Figure 2 Changes in the microstructure pattern of the hippocampal CA1 area in a rat model of TLE after CBX injection. (A,B) Normal microstructure of astrocytes and GJs in the hippocampal CA1 area; (C,D,E,F,G,H) dynamic damage to astrocytes and GJs during epileptogenesis; (I,J) improvement of damaged astrocytes and GJs after the injection of CBX, a Cx43 blocker. The alphabet "A" points to "astrocyte". The black triangle points to an astrocyte, and the long arrow points to a GJ. Scale bars have been shown in each graph. CA1, cornu ammonis 1; TLE, temporal lobe epilepsy; CBX, carbenoxolone; GJ, gap junction; Cx43, connexin 43.

distributed small plaque adjacent to the inner surface of the nuclear membrane, and the nucleolus was occasionally observed. Various kinds of organelles were observed in the cytoplasm. In addition to mitochondria and ERs, abundant GJs, some of which were identified as complex connections with tight junctions, were observed on the membranes of adjacent cells. Twenty-four hours after the onset of $\mathrm{SE}$, the astrocytic nuclear membrane became fuzzy, the nuclear matrix swelled, chromatolysis gradually occurred and heterochromatin was reduced. Large empty areas in the cytoplasm emerged. The number of organelles sharply decreased, and some of them demonstrated vacuolation. The number of GJs on the cell membrane was reduced, or GJs on the cell membrane disappeared (Figure $2 C, D$ ). Fifteen days later, astrocyte proliferation was initiated in the vicinity of neurons and capillaries, and the cellular swelling slowly decreased. The number of GJs increased but was still lower than that in the Con group (Figure $2 E, F)$. On the $60^{\text {th }}$ day after SE, the astrocytes showed typical ultrastructural features of apoptosis. The astrocytes became enlarged again. Astrocytic chromatin condensed in the margin, and the nuclear membrane ruptured or even disappeared. Hypervacuolization of the cytoplasm was maintained (Figure 2G,H). Compared to the SE-60d group, the swelling of the astroglial cell bodies was alleviated in the CBX-60d group. The astrocytic nuclear membrane became distinct and the condensation and margination of the astroglial chromatin was inconspicuous Hypervacuolization of the cytoplasm disappeared (Figure 2I,7).

\section{The effect of CBX on rat hippocampal Cx43 and p-Cx43 protein expression}

Total $\mathrm{Cx} 43$ protein was indicated by a $43 \mathrm{kDa}$ band in Western blot analysis (Figures $3 A$ ). Twenty-four hours after $\mathrm{SE}$, the average ROD for total $\mathrm{Cx} 43$ protein was lower in the SE group than in the Con group and then gradually increased from 7 to 60 days after SE; however, the differences in total $\mathrm{Cx} 43$ protein expression between the Con group and the SE group were not significant using Tukey's post hoc test (Figure 3B). Cx43 protein phosphorylated at Ser368 (p-Cx43) has a molecular weight of $51 \mathrm{kDa}$ (Figures $3 A$ ). Similar to the dynamic expression of total $\mathrm{Cx} 43$ protein, the average ROD for $\mathrm{p}-\mathrm{Cx} 43$ was also significantly lower $24 \mathrm{~h}$ after SE $(\mathrm{P}<0.05$; Figure $3 C)$ before increasing gradually from 7 to 30 days after $\mathrm{SE}$ (both $\mathrm{P}<0.05$ ); the difference in $\mathrm{p}-\mathrm{Cx} 43$ expression compared to the Con group then peaked at 60 days $(\mathrm{P}>0.05)$.

The expression of both total $\mathrm{Cx} 43$ and $\mathrm{p}-\mathrm{Cx} 43$ was lower in all SE and CBX groups than in the corresponding Con 
A

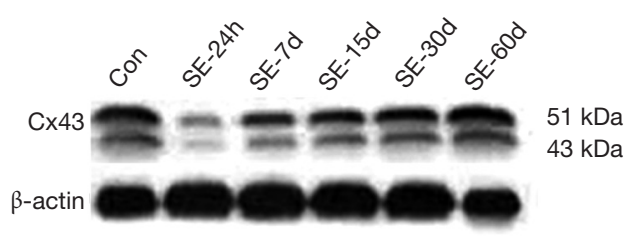

B

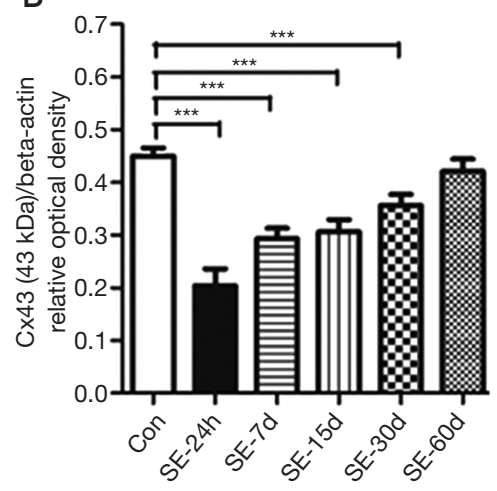

C

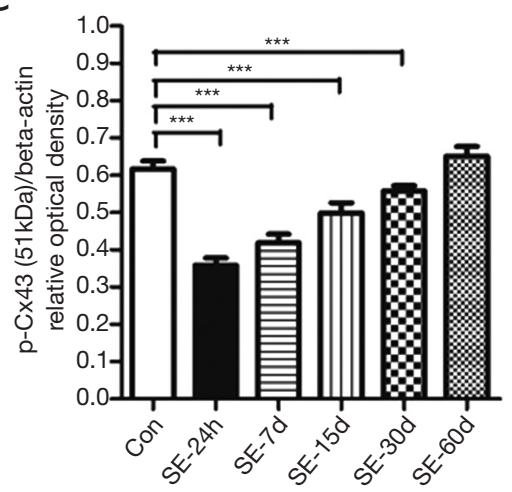

Figure 3 The results of Western blot assays showing the dynamic patterns of $\mathrm{Cx} 43$ and p-Cx43 expression in the hippocampus of a rat model of TLE. (A) Representative results with the corresponding antibodies; (B) the bar graph shows Cx43 protein expression. The ROD for $\mathrm{Cx} 43$ gradually increased until 60 days after SE; (C) the bar graph shows p-Cx43 protein expression. The ROD for p-Cx43 gradually increased until 60 days after SE. $\mathrm{n}=6$ in each subgroup. Compared to SE-60d: *** $\mathrm{P}<0.001$. Cx43, connexin 43; TLE, temporal lobe epilepsy; ROD, relative optical density; SE, status epilepticus.

groups (all $\mathrm{P}<0.05$; Figure $4 A$ ). The expression of total $\mathrm{Cx} 43$ protein was lower in the CBX-15d, CBX-30d and CBX$60 \mathrm{~d}$ groups than in the corresponding SE-15d, SE-30d and SE-60d groups (all $\mathrm{P}<0.05$; Figure $4 B$ ). The $\mathrm{p}-\mathrm{Cx} 43$ protein level was also lower in the CBX-15d, CBX-30d and CBX$60 \mathrm{~d}$ groups than in the corresponding SE-15d, SE-30d and SE-60d groups (all $\mathrm{P}<0.05$; Figure $4 C$ ).

\section{Discussion}

In the present study, astrocytic GJs in a rat model of TLE were observed by TEM following CBX treatment for the first time. SE could inhibit the expression of $\mathrm{Cx} 43$ protein and the formation of GJs in the hippocampi of pilocarpine-induced epileptic rat in the acute phase ( $<7$ days), $C x 43$ protein expression and the number of GJs were higher, and SES appeared to increase in the chronic phase (30-60 days). After continuous injection of the GJ blocker CBX, the expression of $\mathrm{Cx} 43$ protein was lowest in the SE-60d group, followed by a decrease in the number of GJs, and SES attenuation.

GJs electrically and metabolically connect astrocytes to each other. Coupled networks of astrocytes are crucial for the modulation of neuronal activity and synaptic transmission through the clearance and spatial buffering of excess extracellular $\mathrm{K}^{+}$and neurotransmitters (15). The disruption of GJ activity is implicated in epilepsy (16). $\mathrm{SE}$ and seizures cause the uncoupling of astroglial GJs, resulting in gliotransmitter release that contributes to abnormal neuronal activity (17). Furthermore, the reduction or loss of interastrocytic coupling causes the accumulation of $\mathrm{K}^{+}$and glutamate in the extracellular space, which in turn promotes neuronal hyperactivity and SESs (7). Many studies have suggested that major alterations in astrocytic GJs facilitate the generation of seizures and epilepsy (18). Dysfunction of astrocytic GJ has even been demonstrated to cause astrocyte-neuron network hyperexcitability and spontaneous epileptic activity (19). In this study, the abnormal morphology of astrocytic GJs in epileptic rats was observed by TEM, and CBX could reverse this abnormal morphology, but the specific mechanism of this effect should be explored deeply.

$\mathrm{Cx} 43$, which contributes to normal excitatory activity in the CNS, is an important GJ in the hippocampus that supports intercellular communication between neighboring astrocytes (20). The $\mathrm{Cx} 43$ protein was shown to be increased in the hippocampus of the same rat model used in this study not only in the acute phase but also 30-45 days after SE $(21,22)$. Mylvaganam et al. also found that the unphosphorylated form of $\mathrm{Cx} 43$ was increased during $\mathrm{Co}^{2+}$-induced seizures in vitro. A systematic analysis revealed significant changes in the expression of astrocytic Cx43 in an in vitro mouse seizure model (23). Nevertheless, the expression of the $\mathrm{Cx} 43$ protein is still unclear, which may be partly because $\mathrm{Cx} 43$ expression levels do not necessarily reflect the extent of functional coupling since $\mathrm{Cx}$ proteins are tightly regulated. In this study, we observed the decreased expression of the $\mathrm{Cx} 43$ protein along with GJ 
A

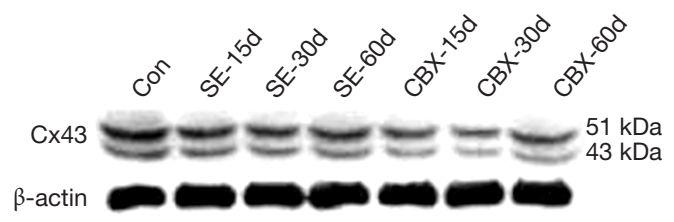

B

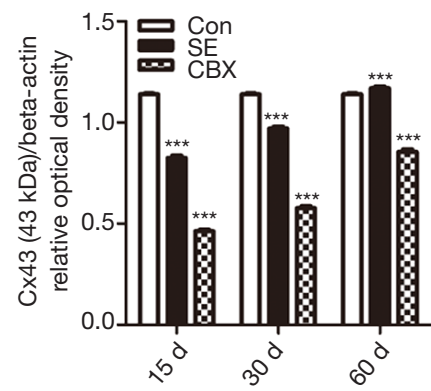

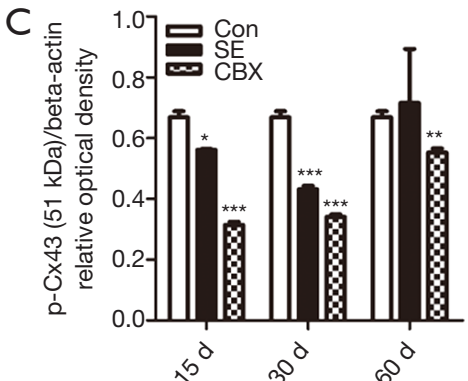

Figure 4 The dynamic patterns of $\mathrm{Cx} 43$ and p-Cx43 expression after CBX injection in the hippocampus of a rat model of TLE. (A) Representative results with the corresponding antibodies; (B) the bar graph shows Cx43 protein expression after CBX injection. The ROD for $\mathrm{Cx} 43$ was lower in the CBX group than in the Con group; $(\mathrm{C})$ the bar graph shows p-Cx43 protein expression after $\mathrm{CBX}$ injection. The ROD for $\mathrm{p}-\mathrm{Cx} 43$ was lower in the CBX group than in the Con group. $\mathrm{n}=6$ in each subgroup. Compared to $\mathrm{SE}-60 \mathrm{~d}$ : *** $\mathrm{P}<0.001,{ }^{* *}, \mathrm{P}<0.01$, *, $\mathrm{P}<0.05$. Cx43, connexin 43; CBX, carbenoxolone; TLE, temporal lobe epilepsy; ROD, relative optical density; Con group, control group; SE, status epilepticus.

dynamics by TEM. Unfortunately, we did not differentiate between $\mathrm{Cx} 43$ derived from astrocytes and $\mathrm{Cx} 43$ derived from other kinds of cells in the hippocampus.

Accruing evidence indicates that phosphorylation of the intracellular tail of $\mathrm{Cx}$ plays a role in gating pore permeability, thereby allowing the gate to dynamically open and close in response to a variety of stimuli (24). $\mathrm{Cx} 43$ possesses at least 21 phosphorylation sites within its cytoplasmic C-terminus that are targeted by a number of kinases, including protein kinase $\mathrm{C}(\mathrm{PKC})$, protein kinase A (PKA), and MAPK. Phosphorylation of Cx43 affects the intercellular communication between astrocytes by altering the gating properties of the channels, the trafficking and degradation of the $\mathrm{Cx} 43$ protein and the assembly/ disassembly of GJs (25). Phosphorylation of the $\mathrm{Cx} 43$ C-terminus at positions S255 and S368 is known to reduce junctional conductance (7). In this study, we found the same expression level of p-Cx43 phosphorylated at S368 as that in a study by Deshpande (19). Furthermore, in the current study, the expression of $\mathrm{p}-\mathrm{Cx} 43$ decreased after $\mathrm{CBX}$ injection in the chronic stage compared to $\mathrm{p}-\mathrm{Cx} 43$ expression in the SE-60d group.

There are still some shortcomings in this study. The expression tendency of the $\mathrm{Cx} 43$ protein from 24 hours to 30 days after SE was inconsistent with those of previous studies $(21,22)$, which may be the result of species differences, different animal models, and the different brain regions investigated as well as differences in seizure duration and time points sampled after the induction of epilepsy. Furthermore, as stated above, the $\mathrm{Cx} 43$ protein was expressed in a mixture of astrocytes, neurons and other kinds of cells in the hippocampus and not only in astrocytes.

In conclusion, the present study demonstrates astrocytic GJ dysfunction as a result of SE and SESs and that CBX can prevent the loss of astrocytic GJs and even improve the morphology of GJs, as shown by TEM. Importantly, our results provide more pathological evidence to understand the molecular mechanisms of astrocyte and GJ dysfunction in TLE, which may play an important role in the pathogenesis of this disorder.

\section{Acknowledgments}

Funding: The National Natural Science Foundation of China provided financial support in the form of direct funding (No: 81601140; 81601141). The sponsor had no role in the design or conduct of this research.

\section{Footnote}

Conflicts of Interest: The authors have no conflicts of interest to declare.

Ethical Statement: The authors are accountable for all aspects of the work in ensuring that questions related to the accuracy or integrity of any part of the work are appropriately investigated and resolved. All applicable international, national, and/or institutional guidelines for the care and use of animals were followed. All procedures performed in studies involving animals were in accordance with the ethical standards of the institution or practice at which the studies were conducted. This article does not 
contain any studies with human participants performed by any of the authors.

\section{References}

1. Perry MS, Duchowny M. Surgical versus medical treatment for refractory epilepsy: outcomes beyond seizure control. Epilepsia 2013;54:2060-70.

2. Vasile F, Dossi E, Rouach N. Human astrocytes: structure and functions in the healthy brain. Brain Struct Funct 2017;222:2017-29.

3. Coulter DA, Steinhäuser C. Role of astrocytes in epilepsy. Cold Spring Harb Perspect Med 2015;5:a022434.

4. Witcher MR, Kirov SA, Harris KM. Plasticity of perisynaptic astroglia during synaptogenesis in the mature rat hippocampus. Glia 2007;55:13-23.

5. Doná F, Conceição IM, Ulrich H, et al. Variations of ATP and its metabolites in the hippocampus of rats subjected to pilocarpine-induced temporal lobe epilepsy. Purinergic Signal 2016;12:295-302.

6. Covelo A, Araque A. Neuronal activity determines distinct gliotransmitter release from a single astrocyte. Elife 2018. doi: 10.7554/eLife.32237.

7. Deshpande T, Li T, Herde MK, et al. Subcellular reorganization and altered phosphorylation of the astrocytic gap junction protein connexin 43 in human and experimental temporal lobe epilepsy. Glia 2017;65:1809-20.

8. Giaume C, Koulakoff A, Roux L, et al. Astroglial networks: a step further in neuroglial and gliovascular interactions. Nat Rev Neurosci 2010;11:87-99.

9. Ran X, Xiang J, Song PP, et al. Effects of gap junctions blockers on fast ripples and connexin in rat hippocampi after status epilepticus. Epilepsy Res 2018;146:28-35.

10. Ventura-Mejía C, Medina-Ceja L. Decreased fast ripples in the hippocampus of rats with spontaneous recurrent seizures treated with carbenoxolone and quinine. Biomed Res Int 2014;2014:282490.

11. Kékesi O, Ioja E, Szabó Z, et al. Recurrent seizurelike events are associated with coupled astroglial synchronization. Front Cell Neurosci 2015;9:215.

12. Sefil F, Arık AE, Acar MD, et al. Interaction between carbenoxolone and valproic acid on pentylenetetrazole kindling model of epilepsy. Int J Clin Exp Med 2015;8:10508-14.

13. Chen W, Gao Z, Ni Y, et al. Carbenoxolone pretreatment and treatment of posttraumatic epilepsy. Neural Regen
Res 2013;8:169-76.

14. Velišsková J. Behavioral characterization of seizures in rats. In: Pitkänen A, Schwartskroin PA, Solomon LM, et al. editors. Models of seizures and epilepsy. Burlington: Elsevier Academic Press, 2006:601-11.

15. Boison D, Steinhäuser C. Epilepsy and astrocyte energy metabolism. Glia 2018;66:1235-43.

16. Manjarrez-Marmolejo J, Franco-Pérez J. Gap junction blockers: an overview of their effects on induced seizures in animal models. Curr Neuropharmacol 2016;14:759-71.

17. Thompson RJ, Jackson MF, Olah ME, et al. Activation of pannexin-1 hemichannels augments aberrant bursting in the hippocampus. Science 2008;322:1555-9.

18. Vargas-Sánchez K, Mogilevskaya M, Rodríguez-Pérez $\mathrm{J}$, et al. Astroglial role in the pathophysiology of status epilepticus: an overview. Oncotarget 2018;9:26954-76.

19. Du M, Li J, Chen L, et al. Astrocytic Kir4.1 channels and gap junctions account for spontaneous epileptic seizure. PLoS Comput Biol 2018;14:e1005877.

20. Torres A, Wang F, Xu Q, et al. Extracellular $\mathrm{Ca}^{2+}$ acts as a mediator of communication from neurons to glia. Sci Signal 2012;5:ra8.

21. Motaghi S, Sayyah M, Babapour V, et al. Hippocampal expression of connexin 36 and connexin 43 during epileptogenesis in pilocarpine model of epilepsy. Iran Biomed J 2017;21:167-73.

22. Mylvaganam S, Zhang L, Wu C, et al. Hippocampal seizures alter the expression of the pannexin and connexin transcriptome. J Neurochem 2010;112:92-102.

23. Lapato AS, Tiwari-Woodruff SK. Connexins and pannexins: at the junction of neuro-glial homeostasis \& disease. J Neurosci Res 2018;96:31-44.

24. Mylvaganam S, Ramani M, Krawczyk M, et al. Roles of gap junctions, connexins, and pannexins in epilepsy. Front Physiol 2014;5:172.

25. Axelsen LN, Calloe K, Holstein-Rathlou NH, et al. Managing the complexity of communication: regulation of gap junctions by post-translational modification. Front Pharmacol 2013;4:130.

Cite this article as: Shu Y, Zhu C, Zeng M, Zhan Q, Hu $\mathrm{Z}, \mathrm{Wu} \mathrm{X}$. The protective effect of carbenoxolone on gap junction damage in the hippocampal CA1 area of a temporal lobe epilepsy rat model. Ann Transl Med 2019;7(22):624. doi: 10.21037/atm.2019.11.04 